\title{
Synthesis, Characterization, and Anti-Inflammatory Activity of Newer Quinazolinone Analogs
}

\author{
Chatrasal Singh Rajput ${ }^{1,2}$ and Shiwani Singhal ${ }^{1}$ \\ ${ }^{1}$ Medicinal Chemistry Division, Department of Pharmacology, L.L.R.M. Medical College, Meerut 250002, India \\ ${ }^{2}$ Jubilant Chemsys Ltd. R \& D B-34, Sector 58, Noida 201301, India \\ Correspondence should be addressed to Chatrasal Singh Rajput; chatrasalrajput@gmail.com
}

Received 24 September 2012; Revised 29 October 2012; Accepted 1 November 2012

Academic Editor: Susana Zacchino

Copyright (c) 2013 C. S. Rajput and S. Singhal. This is an open access article distributed under the Creative Commons Attribution License, which permits unrestricted use, distribution, and reproduction in any medium, provided the original work is properly cited.

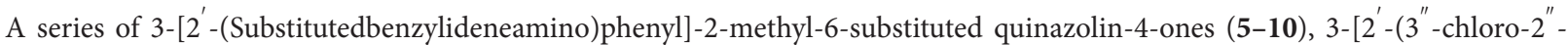
oxo- $4^{\prime \prime}$-substitutedphenylazetidin-1" -yl)phenyl]-2-methyl-6-substitutedquinazolin-4-ones (11-16), and 3-[2'-(2" -substitutedphenyl-4" -oxo-1",3" -thiazolidin-3" -yl)phentl]-2-methyl-6-substitutedquinazolin-4-ones (17-22) have been synthesized in the present study. The structures of the synthesized compounds were assigned on the basis of elemental analysis, IR, ${ }^{1} \mathrm{H}$ NMR, and mass spectral data. All the newly synthesized compounds were screened for anti-inflammatory and analgesic activities.
\end{abstract}

\section{Introduction}

Quinazolinone derivatives represent one of the most active classes of compounds possessing a wide spectrum of biological activity. They are widely used in pharmaceuticals and agrochemicals. Several reports have been published on the biological activities of quinazolinone derivatives, including their anti-inflammatory [1-7], antimalarial [8, 9], antimicrobial, anti-fungal, antibacterial [10-16], anticonvulsant [1720], and antitumor [21, 22] activities. Moreover, large number of quinazolinone derivatives having substitution at 2 and 3 position by different heterocyclic moieties increases antiinflammatory potential of quinazolinone derivatives. Similarly, various azetidinones [23-25] and thiazolidinones [26, 27] have been reported to possess potent anti-inflammatory activity. Looking to the medicinal importance of $4(3 \mathrm{H})$ quinazolinone, 4-thiazolidinone, and azetidinones, we report here the synthesis of a new class of heterocyclic molecules in which all of these moieties are present and try to develop potential bioactive molecules. The structures of the compounds synthesized were assigned on the basis of elemental analysis, IR, ${ }^{1} \mathrm{H}$ NMR, and Mass spectral data. These compounds were evaluated for their anti-inflammatory and analgesic activities.

\section{Materials and Methods}

2.1. Chemistry. The synthetic routes of compounds are outlined in Scheme 1. As shown in Scheme 1, compounds 2-methylsubstitued-4H-3,1-benzoxazin-4-ones (1,2) were synthesized by the known procedure of Bogert [28]. Substituted anthranilic acid reacted with acetic anhydride to give 2-methylsubstituted-4H-3,1-benzoxazin-4-ones $(1,2)$, which further reacted with 2-amino phenyl amine in acetic acid to yield 3-(2'-aminophenyl)-2-methyl-6-substituted quinazolin-4-ones $(3,4)$. Compounds $(3,4)$ when treated with different substituted aromatic aldehydes formed various substituted arylidene derivatives (5-10). These arylidene derivatives on treatment with chloroacetylchloride in presence of triethylamine yielded 3-[2'-( $3^{\prime \prime}$-chloro$2^{\prime \prime}$-oxo-4" -substituted phenylazetidin-1" -yl)phenyl]-2methyl-6-substitutedquinazolin-4-ones (11-16). On the other hand, these arylidene derivatives on reaction with thioglycolic acid and anhydrous $\mathrm{ZnCl}_{2}$ furnished 3-[2'-(2" substitutedphenyl-4" -oxo-1", $3^{\prime \prime}$-thiazolidin-3" -yl)phenyl]2-methyl-6-substitutedquinazolin-4-ones. (17-22). The structures of the compounds synthesized were assigned 

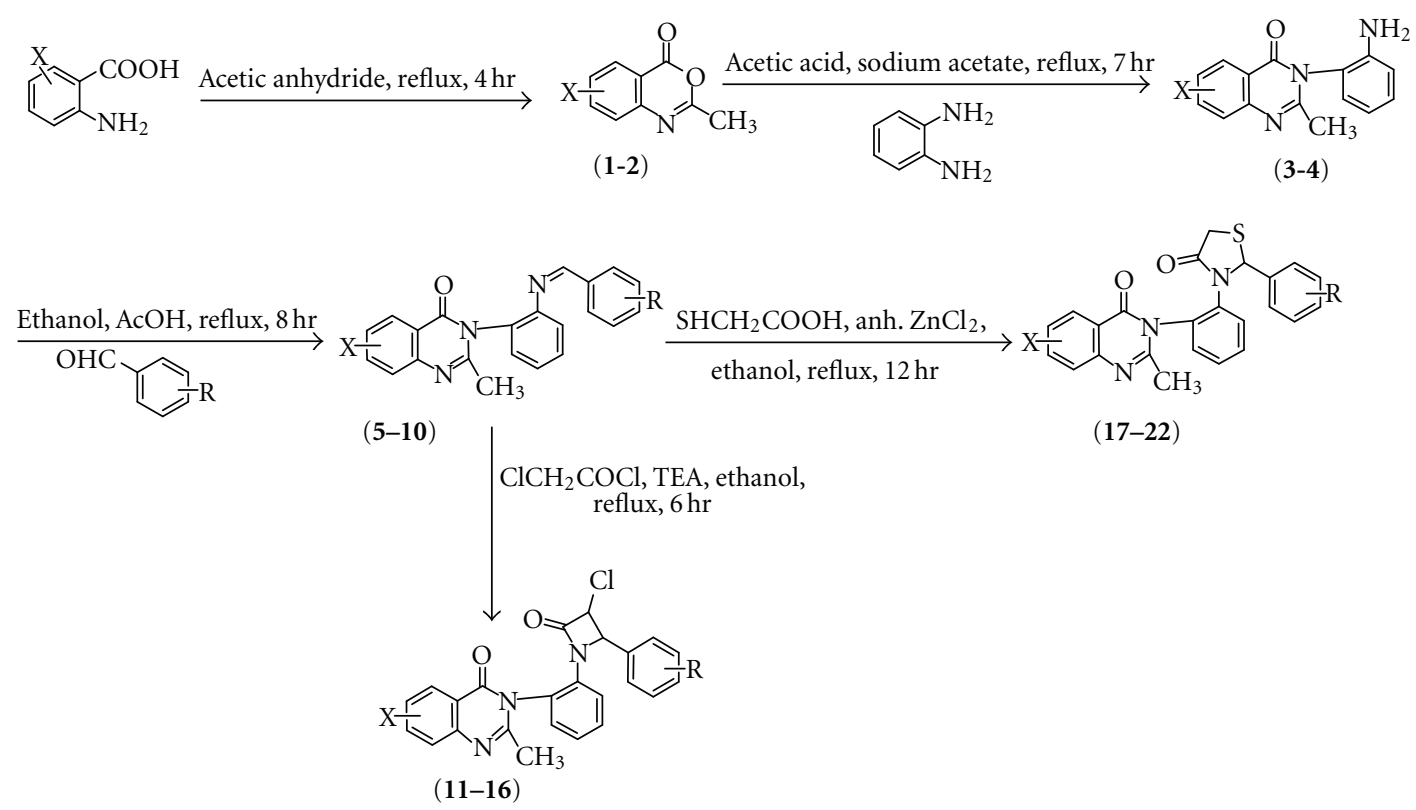

SCHEME 1

on the basis of elemental analysis, IR, ${ }^{1} \mathrm{H}$ NMR, and Mass spectral data.

\subsection{Methods}

2.2.1. Biological Activity. The experiments were performed with albino rats of the Charles-foster stain of either sex, excluding pregnant females, of 70-95 d. weighting 100-150 g. Food (chaw pallet) and water were given to the animals ad libitum. All the compounds were dissolved in propylene glycol. Phenylbutazone was used as reference drug.

Anti-Inflammatory Activity against Carrageenan-Induced Rat's Paw Oedema. This study was done with the procedure of Winter et al. [29]. The rats were divided into three groups (control, treated, and standard drug) of six animals each. A freshly prepared suspension of carrageenan (1\% in $0.9 \%$ saline) $0.05 \mathrm{mn}$ was injected under the planter aponeurosis of the right hind paw of each rat. Test compounds and standard drug were administered orally to the animals of drug-treated groups and the standard drug group, respectively, $1 \mathrm{~h}$ before the carrageenan injection. The paw volume of each rat was measured before 1 and after $3 \mathrm{~h}$ of carrageenan treatment with the help of a plethysmometer. The percent anti-inflammatory activity was calculated according to the formula given below

$$
\text { Percentage of inhibition of oedema }=\left(1-\frac{V_{t}}{V_{c}}\right) \times 100 \text {, }
$$

where $V_{t}$ and $V_{c}$ are the mean increase in paw volume of rats of the treated and the control group, respectively. Results were statistically analyzed.
Analgesic Activity. This activity was assessed following the method of Berkowitz et al. [30]. This method is based on the property of the test compound to antagonize the phenyl quinone-induced pain syndrome in mice. Groups of five mice were injected intraperitoneally with $0.25 \mathrm{~mL}$ of a $0.02 \%$ solution of phenylquinone in ethanol (5\%) $1 \mathrm{~h}$ after of oral administration of the test compound. The number of writhes induced in each mouse was counted for $5 \mathrm{~min}$ (between 5 and $10 \mathrm{~min}$ ) after injection of an irritant. The analgesic effect was expressed as percent protection in comparison to control.

$\%$ protection

$$
\begin{aligned}
= & \left(\frac{1-\text { mean number of writhes in mice of test groups }}{\text { mean number of writhes in mice of control group }}\right) \\
& \times 100 \text {. }
\end{aligned}
$$

2.2.2. Experimental. Melting points were determined in one-end-open capillary tube on Thomas Hoover melting apparatus and are uncorrected. The purities of compounds were checked by thin layer chromatography (TLC) using silica gel-G coated plats. The spots were developed in an iodine chamber and visualized under ultraviolet (UV) lamp. Infrared (IR) absorption spectra were recorded on PerkinElmer RX-1 FTIR spectrometer using potassium bromide (KBr) pellet, and wave numbers were given in $\mathrm{cm}^{-1}$. The ${ }^{1} \mathrm{H}$ NMR spectra were recorded in $\mathrm{CDCl}_{3}$ on a Bruker Avance II $400 \mathrm{NMR}$ spectrometer $(400 \mathrm{MHz})$. The ${ }^{13} \mathrm{C}$ NMR spectra were recorded in $\mathrm{CDCl}_{3}$ on a Bruker Avance II $400 \mathrm{NMR}$ spectrometer operating at $100 \mathrm{MHz}$. The chemical shifts are reported in part per million $(\delta$ ppm) using trimethylsilane (TMS) as an internal standard. Elemental analysis $(\mathrm{C}, \mathrm{H}, \mathrm{N})$ 
was performed on Perkin-Elmer 2400 analyzer, and values were either in $\pm 0.4 \%$ of the calculated.

3-( 2 -Aminophenyl)-2-Methyl-Quinazolin-4-one (3). A mixture of 2-methyl-4H-3,1-benzoxazin-4-one (1) (0.01 mole) with 2 -aminophenylamine $(0.01$ mole $)$ in acetic acid $(40 \mathrm{~mL})$ was refluxed for $7 \mathrm{hr}$. in the presence of sodium acetate. The solvent was removed under reduced pressure. The solid thus obtained was filtered off and recrystallized from toluene to give compound 3 (78\%). m.p. $99^{\circ} \mathrm{C}$. IR $(\mathrm{KBr}) v_{\max }$ in $\mathrm{cm}^{-1}: 3033$ (CH aromatic), 2920 (CH aliphatic), $1690(\mathrm{C}=\mathrm{O})$, $1595(\mathrm{C}=\mathrm{N}) ;{ }^{1} \mathrm{HNMR}\left(\mathrm{CDCl}_{3}\right) \delta$ in ppm: 9.10 (s, 2H, $\mathrm{NH}_{2}$ exchangeable), 6.89-7.61 (m, 8H, Ar-H), 2.29 (s, 3H, $\mathrm{CH}_{3}$ ); Anal. Calcd. for $\mathrm{C}_{15} \mathrm{H}_{13} \mathrm{~N}_{3} \mathrm{O}$ : C, 71.71; $\mathrm{H}, 5.18 ; \mathrm{N}, 16.73$ : Found: C, 71.85; H, 5.20; N, 16.68: MS [M] ${ }^{+}$at $m / z 251$.

The following compound was prepared using a similar procedure described for compound 3.

3-(2'-Aminophenyl)-2-Methyl-6-Bromoquinazolin-4-one (4). (75\%) (Benzene) m.p. $199^{\circ} \mathrm{C}, \mathrm{IR}(\mathrm{KBr}) v_{\max }$ in $\mathrm{cm}^{-1}: 3030$ (CH aromatic), 2925 ( $\mathrm{CH}$ aliphatic), $1695(\mathrm{C}=\mathrm{O}), 1598$ $(\mathrm{C}=\mathrm{N}) ;{ }^{1} \mathrm{H}$ NMR $\left(\mathrm{CDCl}_{3}\right) \delta$ in ppm: $9.06\left(\mathrm{~s}, 2 \mathrm{H}, \mathrm{NH}_{2}\right.$ exchangeable), 6.85-7.51 (m, 7H, $\mathrm{Ar}-\mathrm{H}), 2.24\left(\mathrm{~s}, 3 \mathrm{H}, \mathrm{CH}_{3}\right)$; Anal. Calcd. for $\mathrm{C}_{15} \mathrm{H}_{12} \mathrm{~N}_{3} \mathrm{OBr}$ : C, 54.55; H, 3.64; N, 12.73: Found: C, 54.38; H, 3.65; N, 12.76: MS [M] ${ }^{+}$at $m / z 330$.

3-[ 2 '-(Benzylideneamino)Phenyl]-2-Methyl-Quinazolin-4one ( 5). A solution of compound 3 (0.01 moles) in ethanol $(55 \mathrm{~mL})$ was refluxed with benzaldehyde $(0.01$ moles $)$ separately in the presence of glacial acetic acid $(2.5 \mathrm{~mL})$ for $11 \mathrm{hr}$. The reaction mixture was concentrated, cooled, and then poured into ice water. The separated solid was filtered and recrystallized from methanol to give compound $29(63 \%)$ m.p. $118^{\circ} \mathrm{C}$ : IR $(\mathrm{KBr}) v_{\max }$ in $\mathrm{cm}^{-1}: 3028(\mathrm{CH}$ aromatic), $2930 \mathrm{CH}$ aliphatic), $1705(\mathrm{C}=\mathrm{O}), 1610(\mathrm{C}=\mathrm{N})$; ${ }^{1} \mathrm{HNMR}\left(\mathrm{CDCl}_{3}\right) \delta$ in ppm: 6.79-7.63 (m, 13H, $\left.\mathrm{Ar}-\mathrm{H}\right)$, $6.35(\mathrm{~s}, 1 \mathrm{H}, \mathrm{N}=\mathrm{CH}), 2.21\left(\mathrm{~s}, 3 \mathrm{H}, \mathrm{CH}_{3}\right)$; Anal. Caled. for $\mathrm{C}_{22} \mathrm{H}_{17} \mathrm{~N}_{3} \mathrm{O}$ : C, 77.88; H, 5.01; N, 12.39: Found: C, 77.96; H, 5.00; N, 12.41: MS [M] $]^{+}$at $m / z .339$.

The following compounds were prepared using a similar procedure described for compound $\mathbf{5}$.

3-[2'-(p-Chlorobenzylideneamino)Phenyl]-2-Methyl-Quinazolin-4-one (6). (65\%). (Benzene) m.p. $134^{\circ} \mathrm{C}$ : IR (KBr) $v_{\max }$ in $\mathrm{cm}^{-1}$ : 3028 (CH aromatic), 2929 (CH aliphatic), 1705 $(\mathrm{C}=\mathrm{O}), 1598(\mathrm{C}=\mathrm{N}) 788(\mathrm{C}-\mathrm{Cl}) ;{ }^{1} \mathrm{H}$ NMR $\left(\mathrm{CDCl}_{3}\right) \delta$ in ppm: 6.82-7.65 (m, 12H, Ar-H), 6.38 (s, 1H, N=CH), 2.24 (s, $3 \mathrm{H}, \mathrm{CH}_{3}$ ); Anal. Caled. for $\mathrm{C}_{22} \mathrm{H}_{16} \mathrm{~N}_{3} \mathrm{OCl}$ : C,70.68; $\mathrm{H}$, 4.28; N, 11.24: Found: C, 70.79; H, 4.27; N, 11.26: MS [M] ${ }^{+}$ at $m / z .373 .5$.

3-[2'-(p-Methoxybenzylideneamino)Phenyl]-2-Methyl-Quinazolin-4-one (7). (60\%). (Methanol) m.p. $148^{\circ} \mathrm{C}$ : IR (KBr) $v_{\max }$ in $\mathrm{cm}^{-1}: 3035(\mathrm{CH}$ aromatic), 2932 ( $\mathrm{CH}$ aliphatic), 1700 $(\mathrm{C}=\mathrm{O}), 1600(\mathrm{C}=\mathrm{N}) ;{ }^{1} \mathrm{HNMR}\left(\mathrm{CDCl}_{3}\right) \delta$ in ppm: 6.80-7.61 $(\mathrm{m}, 12 \mathrm{H}, \mathrm{Ar}-\mathrm{H}), 6.40(\mathrm{~s}, 1 \mathrm{H}, \mathrm{N}=\mathrm{CH}), 3.48\left(\mathrm{~s}, 3 \mathrm{H}, \mathrm{OCH}_{3}\right)$ 2.26 (s, 3H, $\mathrm{CH}_{3}$ ); Anal. Caled. for $\mathrm{C}_{23} \mathrm{H}_{19} \mathrm{~N}_{3} \mathrm{O}_{2}$ : C, 74.80; $\mathrm{H}$,
5.15; N, 11.38: Found: C, 74.96; H, 5.17; N, 11.29: MS $[\mathrm{M}]^{+}$ at $m / z .369$.

3-[2'-(Benzylideneamino)Phenyl]-2-Methyl-6-Bromoquinazolin-4-one (8). (64\%). (Ethanol) m.p. $215^{\circ} \mathrm{C}$ : IR (KBr) $v_{\max }$ in $\mathrm{cm}^{-1}: 3033$ ( $\mathrm{CH}$ aromatic), 2926 ( $\mathrm{CH}$ aliphatic), 1698 $(\mathrm{C}=\mathrm{O}), 1606(\mathrm{C}=\mathrm{N}) ;{ }^{1} \mathrm{H} \mathrm{NMR}\left(\mathrm{CDCl}_{3}\right) \delta$ in ppm: 6.83-7.60 (m, 12H, Ar-H), 6.43 (s, 1H, N=CH), 2.22 (s, 3H, $\mathrm{CH}_{3}$ ); Anal. Caled. for $\mathrm{C}_{22} \mathrm{H}_{16} \mathrm{~N}_{3} \mathrm{OBr}$ : C,63.16; H, 3.83; N, 10.05: Found: C, 63.29; H, 3.82; N, 10.07: MS $[\mathrm{M}]^{+}$at $m / z .418$.

3-[2'-(p-Chlorobenzylideneamino)Phenyl]-2-Methyl-6-Bromoquinazolin-4-one (9). (65\%). (Ethanol) m.p. $234^{\circ} \mathrm{C}$ : IR $(\mathrm{KBr}) v_{\max }$ in $\mathrm{cm}^{-1}: 3030(\mathrm{CH}$ aromatic), $2930(\mathrm{CH}$ aliphatic), $1695(\mathrm{C}=\mathrm{O}), 1601(\mathrm{C}=\mathrm{N}), 785(\mathrm{C}-\mathrm{Cl}) ;{ }^{1} \mathrm{HNMR}$ $\left(\mathrm{CDCl}_{3}\right) \delta$ in ppm: $6.81-7.59(\mathrm{~m}, 11 \mathrm{H}, \mathrm{Ar}-\mathrm{H}), 6.41(\mathrm{~s}, 1 \mathrm{H}$, $\mathrm{N}=\mathrm{CH}), 2.25\left(\mathrm{~s}, 3 \mathrm{H}, \mathrm{CH}_{3}\right)$; Anal. Caled. for $\mathrm{C}_{22} \mathrm{H}_{15} \mathrm{~N}_{3} \mathrm{OBrCl}$ : C, 58.34; H, 3.31; N, 9.28: Found: C, 58.55; H, 3.32; N, 9.30: MS $[\mathrm{M}]^{+}$at $m / z .452 .5$.

3-[2'-(p-Methoxybenzylideneamino)Phenyl]-2-Methyl-6Bromoquinazolin-4-one (10). (60\%). (Acetone) m.p. $223^{\circ} \mathrm{C}$ : IR $(\mathrm{KBr}) v_{\max }$ in $\mathrm{cm}^{-1}: 3033(\mathrm{CH}$ aromatic), $2926(\mathrm{CH}$ aliphatic), $1698(\mathrm{C}=\mathrm{O}), 1595(\mathrm{C}=\mathrm{N}) ;{ }^{1} \mathrm{HNMR}\left(\mathrm{CDCl}_{3}\right) \delta$ in ppm: $6.83-7.61(\mathrm{~m}, 11 \mathrm{H}, \mathrm{Ar}-\mathrm{H}), 6.44(\mathrm{~s}, 1 \mathrm{H}, \mathrm{N}=\mathrm{CH})$, $3.44\left(\mathrm{~s}, 3 \mathrm{H}, \mathrm{OCH}_{3}\right) 2.25\left(\mathrm{~s}, 3 \mathrm{H}, \mathrm{CH}_{3}\right)$; Anal. Caled. for $\mathrm{C}_{23} \mathrm{H}_{18} \mathrm{~N}_{3} \mathrm{O}_{2} \mathrm{Br}$ : C, 61.61; H, 4.02; N, 9.38: Found: C, 61.52; $\mathrm{H}, 4.01 ; \mathrm{N}, 9.36: \mathrm{MS}[\mathrm{M}]^{+}$at $m / z .448$

3-[2'-(3"-Chloro-2" -Oxo-4" -Phenylazetidin-1" $-Y l)-$ Phenyl]2-Methyl-Quinazol-In-4-one (11). To a solution of compound $5(0.01$ mole $)$ and triethylamine $(0.5 \mathrm{~mL})$ in ethanol $(55 \mathrm{~mL})$ was added in monochloroacetylchloride (0.014 mole) at $50^{\circ} \mathrm{C}$. The reaction mixture was stirred for $45 \mathrm{~min}$. at room temperature and refluxed for $8 \mathrm{hr}$. The reaction mixture was filtered to removed triethylamine hydrogen chloride and the resultant solution was poured onto crushed ice with constant stirring. The solid thus obtained was recrystallized from acetone to give desired compound 11 (60\%) m.p. $153^{\circ} \mathrm{C}$. IR (KBr): $v_{\max }$ in $\mathrm{cm}^{-1}$ : IR $(\mathrm{KBr}) v_{\max }$ in $\mathrm{cm}^{-1}: 3030$ ( $\mathrm{CH}$ aromatic), 2925 ( $\mathrm{CH}$ aliphatic), $1700(\mathrm{C}=\mathrm{O}), 1595$ $(\mathrm{C}=\mathrm{N}) ;{ }^{1} \mathrm{HNMR}\left(\mathrm{CDCl}_{3}\right) \delta$ in ppm: 6.81-7.98 $(\mathrm{m}, 13 \mathrm{H}$, Ar-H), $6.51(\mathrm{~d}, 1 \mathrm{H}, \mathrm{J}=9.0 \mathrm{~Hz}, \mathrm{CH}-\mathrm{Cl}), 4.60(\mathrm{~d}, 1 \mathrm{H}, \mathrm{J}=$ $9.3 \mathrm{~Hz}, \mathrm{~N}-\mathrm{CH}-\mathrm{Ar}$ ), 2.21 (s, 3H, $\mathrm{CH}_{3}$ ); Anal. Calcd. for $\mathrm{C}_{24} \mathrm{H}_{18} \mathrm{~N}_{3} \mathrm{O}_{2} \mathrm{Cl}$ : C, 69.31; H, 4.33; N, 10.11: Found: C, 69.47; $\mathrm{H}, 4.32$; N, 10.14: MS [M] $]^{+}$at $m / z 415.5$.

The following compounds were prepared using a similar procedure described for compound $\mathbf{1 1}$.

3-[2'-(3" -Chloro-2" -Oxo-4" -(p-Chlorophenyl)Azetidin-

$\left.\left.1^{\prime \prime}-y l\right)-P h e n y l\right]-2-M e t h y l \quad$ Quinazolin-4-one (12). (58\%) (Methanol) m.p. $198^{\circ} \mathrm{C}$. IR $(\mathrm{KBr}): v_{\max }$ in $\mathrm{cm}^{-1}$ : IR $(\mathrm{KBr})$ $v_{\max }$ in $\mathrm{cm}^{-1}: 3033$ ( $\mathrm{CH}$ aromatic), 2928 ( $\mathrm{CH}$ aliphatic), $1705(\mathrm{C}=\mathrm{O}), 1591(\mathrm{C}=\mathrm{N}), 788(\mathrm{C}-\mathrm{Cl}) ;{ }^{1} \mathrm{HNMR}\left(\mathrm{CDCl}_{3}\right) \delta$ in ppm: $6.83-7.95(\mathrm{~m}, 12 \mathrm{H}, \mathrm{Ar}-\mathrm{H}), 6.56(\mathrm{~d}, 1 \mathrm{H}, \mathrm{J}=8.8 \mathrm{~Hz}$, $\mathrm{CH}-\mathrm{Cl}), 4.62$ (d, 1H, J = 9.0 Hz, N-CH-Ar), 2.25 (s, 3H, $\mathrm{CH}_{3}$ );. Anal. Calcd. for $\mathrm{C}_{24} \mathrm{H}_{17} \mathrm{~N}_{3} \mathrm{O}_{2} \mathrm{Cl}_{2}$ : C, 64.00; $\mathrm{H}, 3.78$; 
TABLE 1: Anti-inflammatory and analgesic data of compounds 5-22.

\begin{tabular}{|c|c|c|c|}
\hline Comp. number & $\begin{array}{c}\text { Dose } \\
\text { (mg/kg p.o.) }\end{array}$ & $\begin{array}{c}\text { Anti-inflammatory activity \% } \\
\text { oedema inhibition }\end{array}$ & Analgesic activity $\%$ \\
\hline 5 & 50 & 15.1 & 11.6 \\
\hline 6 & 50 & 21.8 & 16.2 \\
\hline 7 & 50 & 16.7 & 14.9 \\
\hline 8 & 50 & 18.2 & 17.1 \\
\hline 9 & 50 & 24.1 & 19.3 \\
\hline 10 & 50 & 20.4 & 18.5 \\
\hline 11 & 50 & 18.3 & 14.6 \\
\hline 12 & 50 & 24.6 & 18.7 \\
\hline 13 & 50 & 20.8 & 16.2 \\
\hline 14 & 50 & 22.4 & 20.6 \\
\hline 15 & 50 & 27.3 & 25.8 \\
\hline 16 & 50 & 26.1 & 22.2 \\
\hline 17 & 50 & 22.9 & 16.3 \\
\hline 18 & 50 & 26.3 & 19.8 \\
\hline 19 & 50 & 24.5 & 22.4 \\
\hline 20 & 50 & 27.1 & 25.1 \\
\hline 21 & 50 & 32.5 & 29.6 \\
\hline 22 & 50 & 29.8 & 28.3 \\
\hline Phenylbutazone & 50 & 38.8 & 36.5 \\
\hline
\end{tabular}

N, 9.33: Found: C, 64.15; H, 3.80; N, 9.30: MS $[\mathrm{M}]^{+}$at $m / z$ 450.

3- $22^{\prime}-\left(3^{\prime \prime}-\right.$ Chloro- $2^{\prime \prime}-O x o-4^{\prime \prime}-(p-M e t h o x y p h e n y l)$ Azetidin-

$1^{\prime \prime}$-yl)-Phenyl]-2-Methyl Quinazolin-4-one (13). (56\%) (Ethanol)m.p. $212^{\circ} \mathrm{C}$. IR (KBr): $v_{\max }$ in $\mathrm{cm}^{-1}$ : IR (KBr) $v_{\max }$ in $\mathrm{cm}^{-1}: 3028$ (CH aromatic), 2930 ( $\mathrm{CH}$ aliphatic), 1700 $(\mathrm{C}=\mathrm{O}), 1595(\mathrm{C}=\mathrm{N}) ;{ }^{1} \mathrm{HNMR}\left(\mathrm{CDCl}_{3}\right) \delta$ in ppm: 6.81-7.93 $(\mathrm{m}, 12 \mathrm{H}, \mathrm{Ar}-\mathrm{H}), 6.54(\mathrm{~d}, 1 \mathrm{H}, \mathrm{J}=9.0 \mathrm{~Hz}, \mathrm{CH}-\mathrm{Cl}), 4.65$ (d, $1 \mathrm{H}, \mathrm{J}=9.3 \mathrm{~Hz}, \mathrm{~N}-\mathrm{CH}-\mathrm{Ar}), 3.47$ (s, 3H, $\left.\mathrm{OCH}_{3}\right) 2.23$ (s, 3H, $\mathrm{CH}_{3}$ ); Anal. Calcd. for $\mathrm{C}_{25} \mathrm{H}_{20} \mathrm{~N}_{3} \mathrm{O}_{3} \mathrm{Cl}$ : C, 67.34; $\mathrm{H}, 4.49 ; \mathrm{N}$, 9.43: Found: C, 67.15; H, 4.50; N, 9.40: MS [M] ${ }^{+}$at $m / z 445.5$.

3-[2'-(3"-Chloro-2" -Oxo-4" -Phenylazetidin-1" $-y l)-$ Phenyl]2-Methyl-6-Bromo-Quinazolin-4-one (14). (58\%) (Methanol) m.p. $176^{\circ} \mathrm{C}$. IR $(\mathrm{KBr}): v_{\max }$ in $\mathrm{cm}^{-1}$ : IR $(\mathrm{KBr}) v_{\max }$ in $\mathrm{cm}^{-1}: 3030$ (CH aromatic), 2925 (CH aliphatic), 1695 $(\mathrm{C}=\mathrm{O}), 1595(\mathrm{C}=\mathrm{N}) ;{ }^{1} \mathrm{HNMR}\left(\mathrm{CDCl}_{3}\right) \delta$ in ppm: 6.80-7.92 $(\mathrm{m}, 12 \mathrm{H}, \mathrm{Ar}-\mathrm{H}), 6.54(\mathrm{~d}, 1 \mathrm{H}, \mathrm{J}=9.2 \mathrm{~Hz}, \mathrm{CH}-\mathrm{Cl}), 4.64$ (d, $1 \mathrm{H}, \mathrm{J}=9.1 \mathrm{~Hz}, \mathrm{~N}-\mathrm{CH}-\mathrm{Ar}$ ), $2.22\left(\mathrm{~s}, 3 \mathrm{H}, \mathrm{CH}_{3}\right)$;. Anal. Calcd. for $\mathrm{C}_{24} \mathrm{H}_{17} \mathrm{~N}_{3} \mathrm{O}_{2} \mathrm{ClBr}$ : C, 58.24; $\mathrm{H}, 3.44 ; \mathrm{N}, 8.49$ : Found: $\mathrm{C}$, 58.36; $\mathrm{H}, 3.43 ; \mathrm{N}, 8.51: \mathrm{MS}[\mathrm{M}]^{+}$at $m / z$ 494.5.

3-[2'-(3" $3^{\prime \prime}$ Chloro-2" -Oxo-4" -(p-Chlorophenyl)Azetidin-1" yl)-Phenyl]-2-Methyl-6-Bromo Quinazolin-4-one (15). (54\%) (Ethanol) m.p. $204^{\circ} \mathrm{C}$. IR (KBr): $v_{\max }$ in $\mathrm{cm}^{-1}$ : IR (KBr) $v_{\max }$ in $\mathrm{cm}^{-1}: 3027$ (CH aromatic), 2930 ( $\mathrm{CH}$ aliphatic), 1710 $(\mathrm{C}=\mathrm{O}), 1598(\mathrm{C}=\mathrm{N}), 785(\mathrm{C}-\mathrm{Cl}) ;{ }^{1} \mathrm{HNMR}\left(\mathrm{CDCl}_{3}\right) \delta$ in ppm: 6.79-7.90 (m, 11H, Ar-H), $6.55(\mathrm{~d}, 1 \mathrm{H}, \mathrm{J}=9.3 \mathrm{~Hz}$, $\mathrm{CH}-\mathrm{Cl}), 4.61$ (d, $1 \mathrm{H}, \mathrm{J}=8.8 \mathrm{~Hz}, \mathrm{~N}-\mathrm{CH}-\mathrm{Ar}), 2.20$ (s, 3H,
$\mathrm{CH}_{3}$ );. Anal. Calcd. for $\mathrm{C}_{24} \mathrm{H}_{16} \mathrm{~N}_{3} \mathrm{O}_{2} \mathrm{Cl}_{2} \mathrm{Br}$ : C, 54.44; $\mathrm{H}, 3.02$; N, 7.94: Found: C, 54.61; H, 3.01; N, 7.95: MS [M] ${ }^{+}$at $m / z$ 529.

3- $\left[2^{\prime}-\left(3^{\prime \prime}-\right.\right.$ Chloro- $2^{\prime \prime}-O x o-4^{\prime \prime}-(P-M e t h o x y p h e n y l)$ Azetidin-

$\left.\left.1^{\prime \prime}-y l\right)-P h e n y l\right]-2-M e t h y l \quad Q u i n a z o l i n-4-o n e \quad(16) . \quad(60 \%)$ (Acetone) m.p. $246^{\circ}$ C. IR (KBr): $v_{\max }$ in $\mathrm{cm}^{-1}$ : IR (KBr) $v_{\max }$ in $\mathrm{cm}^{-1}: 3030$ ( $\mathrm{CH}$ aromatic), 2930 ( $\mathrm{CH}$ aliphatic), 1700 $(\mathrm{C}=\mathrm{O}), 1590(\mathrm{C}=\mathrm{N}) ;{ }^{1} \mathrm{HNMR}\left(\mathrm{CDCl}_{3}\right) \delta$ in ppm: 6.80-7.92 (m, 11H, Ar-H), 6.52 (d, 1H, J = 9.2 Hz, CH-Cl), 4.62 (d, $1 \mathrm{H}, \mathrm{J}=9.0 \mathrm{~Hz}, \mathrm{~N}-\mathrm{CH}-\mathrm{Ar}), 3.49\left(\mathrm{~s}, 3 \mathrm{H}, \mathrm{OCH}_{3}\right) 2.25(\mathrm{~s}, 3 \mathrm{H}$, $\mathrm{CH}_{3}$ ); . Anal. Calcd. for $\mathrm{C}_{25} \mathrm{H}_{19} \mathrm{~N}_{3} \mathrm{O}_{3} \mathrm{ClBr}$ : C, 57.20; H, 3.62; $\mathrm{N}$, 8.01: Found: C, 57.31; H, 3.61; N, 8.06: MS [M] $]^{+}$at $m / z$ 524.5 .

3-[2'-(2" -Phenyl-4" -Oxo-1", $3^{\prime \prime}-$ Thiazolidin-3" $\left.-y l\right)$ Phenyl $]-2-$ Methyl-6-Quinazolin-4-one (17). A mixture of compound 5(0.01 moles), thioglycolic acid (0.012 moles) in absolute dioxane $(75 \mathrm{~mL})$ and in the presence of anhydrous $\mathrm{ZnCl}_{2}$ was refluxed for $18 \mathrm{hr}$. The solvent was removed under reduced pressure. The solid thus obtained was filtered and washed with water. The product finally obtained was dried and recrystallized from ethanol to yield compound 17 (55\%) m.p. $164^{\circ} \mathrm{C}$. IR (KBr): $v_{\max }$ in $\mathrm{cm}^{-1}:$ IR $(\mathrm{KBr}) v_{\max }$ in $\mathrm{cm}^{-1:} 3032$ ( $\mathrm{CH}$ aromatic), 2931 ( $\mathrm{CH}$ aliphatic), $1705(\mathrm{C}=\mathrm{O}), 1595$ $(\mathrm{C}=\mathrm{N}), 685(\mathrm{C}-\mathrm{S}-\mathrm{C}) ;{ }^{1} \mathrm{HNMR}\left(\mathrm{CDCl}_{3}\right) \delta$ in ppm: 6.80-7.97 (m, 13H, Ar-H), 4.55 (s, 1H, N-CH-Ar), 4.25 (s, 2H, $\mathrm{CH}_{2}$ ) 2.25 (s, 3H, $\mathrm{CH}_{3}$ ); Anal. Calcd. for $\mathrm{C}_{24} \mathrm{H}_{19} \mathrm{~N}_{3} \mathrm{O}_{2} \mathrm{~S}$ : C, 69.73; H, 4.60; N, 10.17: Found: C, 69.82; H, 4.61; N, 10.22: MS $[\mathrm{M}]^{+}$at $m / z 413$. 
3-[2'-(2" $-(p-C h l o r o p h e n y l)-4^{\prime \prime}-O x o-1^{\prime \prime}, \quad 3{ }^{\prime \prime}-$ Thiazolidin-

$3^{\prime \prime}$-Yl)Phenyl]-2-Methyl-Quinazolin-4-one. (18). (49\%)

(Methanol) m.p. $192^{\circ} \mathrm{C}$. IR (KBr): $v_{\max }$ in $\mathrm{cm}^{-1}$ : IR $(\mathrm{KBr})$ $v_{\max }$ in $\mathrm{cm}^{-1}: 3030$ ( $\mathrm{CH}$ aromatic), 2930 ( $\mathrm{CH}$ aliphatic), 1700 $(\mathrm{C}=\mathrm{O}), 1598(\mathrm{C}=\mathrm{N}), 784(\mathrm{C}-\mathrm{Cl}), 688(\mathrm{C}-\mathrm{S}-\mathrm{C}) ;{ }^{1} \mathrm{HNMR}$ $\left(\mathrm{CDCl}_{3}\right) \delta$ in ppm: 6.83-7.96 (m, 12H, Ar-H), $4.58(\mathrm{~s}, 1 \mathrm{H}$, $\mathrm{N}-\mathrm{CH}-\mathrm{Ar}), 4.21\left(\mathrm{~s}, 2 \mathrm{H}, \mathrm{CH}_{2}\right) 2.22\left(\mathrm{~s}, 3 \mathrm{H}, \mathrm{CH}_{3}\right)$;. Anal. Calcd. for $\mathrm{C}_{24} \mathrm{H}_{18} \mathrm{~N}_{3} \mathrm{O}_{2} \mathrm{SCl}$ : C, 64.36; H, 4.02; N, 9.39: Found: $\mathrm{C}, 64.41 ; \mathrm{H}, 4.03 ; \mathrm{N}, 9.35$ : MS $[\mathrm{M}]^{+}$at $m / z$ 447.5.

3-[2'-(2" -(p-Methoxyphenyl)-4" -Oxo-1", $3^{\prime \prime}-$ Thiazolidin-3" yl)Phenyl]-2-Methyl Quinazolin-4-one (19). (50\%) (Acetone) m.p. $204^{\circ} \mathrm{C}$. IR (KBr): $v_{\max }$ in $\mathrm{cm}^{-1}$ : IR $(\mathrm{KBr}) v_{\max }$ in $\mathrm{cm}^{-1}: 3032$ (CH aromatic), 2927 ( $\mathrm{CH}$ aliphatic), $1700(\mathrm{C}=\mathrm{O})$, $1600(\mathrm{C}=\mathrm{N}), 690(\mathrm{C}-\mathrm{S}-\mathrm{C}),{ }^{1} \mathrm{HNMR}\left(\mathrm{CDCl}_{3}\right) \delta$ in ppm: 6.81-7.95 (m, 12H, Ar-H), 4.52 (s, 1H, N-CHvAr), 4.23 (s, $\left.2 \mathrm{H}, \mathrm{CH}_{2}\right) 3.51\left(\mathrm{~s}, 3 \mathrm{H}, \mathrm{OCH}_{3}\right), 2.20\left(\mathrm{~s}, 3 \mathrm{H}, \mathrm{CH}_{3}\right)$;. Anal. Calcd. for $\mathrm{C}_{25} \mathrm{H}_{21} \mathrm{~N}_{3} \mathrm{O}_{3} \mathrm{~S}$ : C, 67.72; H, 4.74; N, 9.48: Found: C,67.88; $\mathrm{H}, 4.73 ; \mathrm{N}, 9.51: \mathrm{MS}[\mathrm{M}]^{+}$at $m / z 443$.

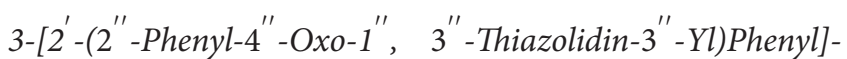
2-Methyl-6-Bromo Quinazolin-4-one (20). (55\%) (Ethanol) m.p. $236^{\circ} \mathrm{C}$. IR (KBr): $v_{\max }$ in $\mathrm{cm}^{-1}$ : IR $(\mathrm{KBr}) v_{\max }$ in $\mathrm{cm}^{-1}$ : 3028 (CH aromatic), 2933 ( $\mathrm{CH}$ aliphatic), 1705 (C=O), 1600 $(\mathrm{C}=\mathrm{N}), 690(\mathrm{C}-\mathrm{S}-\mathrm{C}) ;{ }^{1} \mathrm{H}$ NMR $\left(\mathrm{CDCl}_{3}\right) \delta$ in ppm: 6.81-7.94 (m, 12H, Ar-H), 4.56 (s, 1H, N-CH-Ar), 4.22 (s, 2H, $\mathrm{CH}_{2}$ ) $2.24\left(\mathrm{~s}, 3 \mathrm{H}, \mathrm{CH}_{3}\right)$. Anal. Calcd. for $\mathrm{C}_{24} \mathrm{H}_{18} \mathrm{~N}_{3} \mathrm{O}_{2} \mathrm{SBr}$ : C, 58.54; H, 3.66; N, 8.54: Found: C,58.66; H, 3.67; N, 8.56: MS $[\mathrm{M}]^{+}$at $m / z 492.5$.

3-[2'-(2" -(p-Chlorophenyl)-4" -Oxo-1", $\quad 3^{\prime \prime}-$ Thiazolidin-3" yl)Phenyl]-2-Methyl-6-Bromo Quinazolin-4-one (21). (48\%) (Methanol) m.p. $267^{\circ} \mathrm{C}$. IR (KBr): $v_{\max }$ in $\mathrm{cm}^{-1}$ : IR (KBr) $v_{\max }$ in $\mathrm{cm}^{-1}: 3033$ (CH aromatic), 2925 ( $\mathrm{CH}$ aliphatic), 1705 $(\mathrm{C}=\mathrm{O}), 1595(\mathrm{C}=\mathrm{N}), 784(\mathrm{C}-\mathrm{Cl}), 685(\mathrm{C}-\mathrm{S}-\mathrm{C}) ;{ }^{1} \mathrm{HNMR}$ $\left(\mathrm{CDCl}_{3}\right) \delta$ in ppm: 6.80-7.93 (m, 11H, Ar-H), $4.52(\mathrm{~s}, 1 \mathrm{H}$, $\mathrm{N}-\mathrm{CH}-\mathrm{Ar}), 4.24\left(\mathrm{~s}, 2 \mathrm{H}, \mathrm{CH}_{2}\right) 2.26\left(\mathrm{~s}, 3 \mathrm{H}, \mathrm{CH}_{3}\right)$;. Anal. Calcd. for $\mathrm{C}_{24} \mathrm{H}_{17} \mathrm{~N}_{3} \mathrm{O}_{2}$ SClBr: C,54.70; $\mathrm{H}, 3.23 ; \mathrm{N}, 7.98$ : Found: C, 54.86; H, 3.22; N, 7.97: MS [M] ${ }^{+}$at $m / z$ 526.5.

3-[2'-(2"-(p-Methoxyphenyl)-4"-Oxo-1", $3^{\prime \prime}-$ Thiazolidin-3"yl)Phenyl]-2-Methyl-6-Bromo Quinazolin-4-one (22). (55\%) (Ethanol) m.p. $254^{\circ} \mathrm{C}$. IR (KBr): $v_{\max }$ in $\mathrm{cm}^{-1}: \mathrm{IR}(\mathrm{KBr}) v_{\max }$ in $\mathrm{cm}^{-1}: 3031$ ( $\mathrm{CH}$ aromatic), 2928 ( $\mathrm{CH}$ aliphatic), 1710 $(\mathrm{C}=\mathrm{O}), 1605(\mathrm{C}=\mathrm{N}), 685(\mathrm{C}-\mathrm{S}-\mathrm{C}) ;{ }^{1} \mathrm{HNMR}\left(\mathrm{CDCl}_{3}\right) \delta$ in ppm: 6.81-7.92 (m, 11H, Ar-H), 4.59 (s,1H, N-CH-Ar), 4.25 (s, $\left.2 \mathrm{H}, \mathrm{CH}_{2}\right), 3.49$ (s, $\left.3 \mathrm{H}, \mathrm{OCH}_{3}\right), 2.26$ (s, 3H, $\left.\mathrm{CH}_{3}\right)$;. Anal. Calcd. for $\mathrm{C}_{25} \mathrm{H}_{20} \mathrm{~N}_{3} \mathrm{O}_{3} \mathrm{SBr}$ : C, 57.47; H, 3.83; N, 8.05: Found: C, 57.62; H, 3.82; N, 8.03: MS [M] ${ }^{+}$at $m / z 522$.

\section{Results and Discussion}

3.1. Anti-Inflammatory Activity. The anti-inflammatory activity of newly synthesis compounds is showed in Table 1. All the compounds (5-22) were screened for antiinflammatory activity at $50 \mathrm{mg} / \mathrm{kg}$ p.o. using phenylbutazone as reference drug. All the tested compounds exhibited 15.1 to $32.5 \%$ edema inhibition at the dose of $50 \mathrm{mg} / \mathrm{kg}$ p.o. When the compounds were substituted with $p$-chlorophenyl group showed better anti-inflammatory activity than phenyl group. Compounds (5-10) showed anti-inflammatory from 15.1 to $20.4 \%$. Compound 9, which was substituted by $2^{\prime}$-(p-chlorobenzylideneamino)phenyl group at the IIIrd position of quinazolinone moiety showed better antiinflammatory activity (20.4\%) than compounds 5, 6, 7, 8, and 10. Cyclizations of these compounds into azetidinones and thiazolidinones have shown better anti-inflammatory activity than corresponding arylidene derivatives. Azetidinones (11-16) and thiazolidinones (17-22) have shown the antiinflammatory activity from (24.6-27.3\%) and (22.9-32.5\%) respectively. In azetidinones, compound $\mathbf{1 5}$ showed better anti-inflammatory activity at the dose of $50 \mathrm{mg} / \mathrm{kg}$ p.o. than compounds 11, 12, 13, 14, and 16. Compound 11 exhibited lower percentage inhibition of oedema $(24.6 \%)$ among azetidinones. Thiazolidinones (17-22) generally showed better anti-inflammatory then corresponding azetidinones (11-16). Among all the tested compounds, 3 - $\left[2^{\prime}-\left(2^{\prime \prime}-(\mathrm{p}\right.\right.$-chlorophenyl $)-4^{\prime \prime}$-oxo-1 ${ }^{\prime \prime}, 3^{\prime \prime}$-thiazolidin-3" yl)phenyl]-2-methyl-6-bromo quinazolin-4-one (21) showed better anti-inflammatory activity (32.5\%).

3.2. Analgesic Activity. Compounds (5-10) have shown moderate analgesic activity (11.6-19.3\%). The compound 9 showed good analgesic activity (19.3\%) then compounds 5, 6, 7,8 , and 10 . The formation of azetidinones (11-16) and thiazolidinones (17-22) from compounds (5-10) showed better analgesic activity than corresponding arylidene derivatives. Among these, compound 21 showed the better analgesic activity (29.6\%) than other tested compounds. The results of the biological study are depicted in Table 1.

\section{Conclusion}

It may be concluded that the compounds having 4chlorophenyl group of quinazolinone moiety showed better anti-inflammatory activity than phenyl moiety. Azetidinone and thiazolidinone derivatives were found active than corresponding schiff base derivatives. Further, thiazolidinones showed better anti-inflammatory and analgesics activities in comparison to their corresponding azetidinones. Among all the tested compounds, compound 21 showed better antiinflammatory (32.5\%) and analgesics (29.6\%) activities than other compounds of series.

\section{Acknowledgment}

This paper is dedicated to late Professor Ashok Kumar.

\section{References}

[1] A. Kumar, S. Sharma, Archana et al., "Some new 2,3,6trisubstituted quinazolinones as potent anti-inflammatory, analgesic and COX-II inhibitors," Bioorganic and Medicinal Chemistry, vol. 11, no. 23, pp. 5293-5299, 2003. 
[2] B. Maggio, G. Daidone, D. Raffa et al., "Synthesis and pharmacological study of ethyl 1-methyl-5-(substituted 3,4-dihydro-4oxoquinazolin-3-yl)-1H-pyrazole-4-acetates," European Journal of Medicinal Chemistry, vol. 36, no. 9, pp. 737-742, 2001.

[3] R. S. Giri, H. M. Thaker, T. Giordano et al., "Design, synthesis and characterization of novel 2-(2,4-disubstituted-thiazole-5yl)-3-aryl-3H-quinazoline-4-one derivatives as inhibitors of NF- $\kappa \mathrm{B}$ and AP-1 mediated transcription activation and as potential anti-inflammatory agents," European Journal of Medicinal Chemistry, vol. 44, no. 5, pp. 2184-2189, 2009.

[4] E. Manivannan and S. C. Chaturvedi, "Analogue-based design, synthesis and molecular docking analysis of 2,3-diaryl quinazolinones as non-ulcerogenic anti-inflammatory agents," Bioorganic and Medicinal Chemistry, vol. 19, no. 15, pp. 4520-4528, 2011.

[5] A. Kumar, C. S. Rajput, and S. K. Bhati, "Synthesis of 3-[4' (p-chlorophenyl)-thiazol-2' -yl]-2-[(substituted azetidinone/ thiazolidinone)-aminomethyl]-6-bromoquinazolin-4-ones as anti-inflammatory agent," Bioorganic and Medicinal Chemistry, vol. 15, no. 8, pp. 3089-3096, 2007.

[6] R. S. Giri, H. M. Thaker, T. Giordano et al., "Design, synthesis and evaluation of novel 2-thiophen-5-yl-3H-quinazolin-4-one analogues as inhibitors of transcription factors NF- $\kappa$ B and AP1 mediated transcriptional activation: their possible utilization as anti-inflammatory and anti-cancer agents," Bioorganic and Medicinal Chemistry, vol. 18, no. 7, pp. 2796-2808, 2010.

[7] E. Bansal, V. K. Srivastava, and A. Kumar, "Synthesis and anti-inflammatory activity of 1 -acetyl-5-substitute daryl-3- $(\beta$ aminonaphthyl)-2-pyrazolines and $\beta$-(substitute daminoethyl) amidonaphthalenes," European Journal of Medicinal Chemistry, vol. 36, no. 1, pp. 81-92, 2001.

[8] S. Zhu, J. Wang, G. Chandrashekar, E. Smith, X. Liu, and Y. Zhang, "Synthesis and evaluation of 4-quinazolinone compounds as potential antimalarial agents," European Journal of Medicinal Chemistry, vol. 45, no. 9, pp. 3864-3869, 2010.

[9] S. Zhu, Q. Zhang, C. Gudise, L. Wei, E. Smith, and Y. Zeng, "Synthesis and biological evaluation of febrifugine analogues as potential antimalarial agents," Bioorganic and Medicinal Chemistry, vol. 17, no. 13, pp. 4496-4502, 2009.

[10] G. P. Suresha, R. Suhas, W. Kapfo, and D. Channe Gowda, "Urea/thiourea derivatives of quinazolinone-lysine conjugates: synthesis and structure-activity relationships of a new series of antimicrobials," European Journal of Medicinal Chemistry, vol. 46, no. 6, pp. 2530-2540, 2011.

[11] M. S. Mohameda, M. M. Kamel, E. M. Kassem, N. Abotaleb, S. I. AbdEl-Moez, and M. F. Ahmed, "Novel 6,8-dibromo$4(3 H)$ quinazolinone derivatives of anti-bacterial and antifungalactivities," European Journal of Medicinal Chemistry, vol. 45, no. 8, pp. 3311-3319, 2010.

[12] D. R. Patel and K. C. Patel, "Synthesis, antimicrobial activity and application of some novel quinazolinone based monoazo reactive dyes on various fibres," Dyes and Pigments, vol. 90, no. 1, pp. 1-10, 2011.

[13] D. Kohli, S. R. Hashim, S. Vishal, M. Sharma, and A. K. Simgh, "Synthesis and antibacterial activity of quinazolinone derivatives," International Journal of Pharmacy and Pharmaceutical Sciences, vol. 1, no. 1, pp. 163-169, 2009.

[14] N. B. Patel and J. C. Patel, "Synthesis and antimicrobial activity of Schiff bases and 2-azetidinones derived from quinazolin4(3H)-one," Arabian Journal of Chemistry, vol. 4, no. 4, pp. 403-411, 2011.
[15] S. N. Pandeya, D. Sriram, G. Nath, and E. De Clercq, "Synthesis, antibacterial, antifungal and anti-HIV evaluation of Schiff and Mannich bases of isatin derivatives with 3-amino2-methylmercapto quinazolin-4(3H)-one," Pharmaceutica Acta Helvetiae, vol. 74, no. 1, pp. 11-17, 1999.

[16] A. Kumar, P. Sharma, P. Kumari, and B. Lal Kalal, "Exploration of antimicrobial and antioxidant potential of newly synthesized 2,3-disubstituted quinazoline-4(3H)-ones," Bioorganic and Medicinal Chemistry Letters, vol. 21, no. 14, pp. 4353-4357, 2011.

[17] M. Zappalà, S. Grasso, N. Micale et al., "1-Aryl-6,7methylenedioxy- $3 \mathrm{H}$-quinazolin-4-ones as anticonvulsant agents," Bioorganic and Medicinal Chemistry Letters, vol. 13, no. 24, pp. 4427-4430, 2003.

[18] V. Jatav, P. Mishra, S. Kashaw, and J. P. Stables, "CNS depressant and anticonvulsant activities of some novel 3-[5substituted 1,3,4-thiadiazole-2-yl]-2-styryl quinazoline-4(3H)ones," European Journal of Medicinal Chemistry, vol. 43, no. 9, pp. 1945-1954, 2008.

[19] A. S. El-Azab and K. E. H. ElTahir, "Synthesis and anticonvulsant evaluation of some new 2, 3, 8-trisubstituted-4(3H)quinazoline derivatives," Bioorganic \& Medicinal Chemistry Letters, vol. 22, no. 1, pp. 327-333, 2012.

[20] S. K. Kashaw, V. Kashaw, P. Mishra, N. K. Jain, and J. P. Stables, "Synthesis, anticonvulsant and CNS depressant activity of some new bioactive 1-(4-substituted-phenyl)-3-(4-oxo-2phenyl/ethyl-4H-quinazolin-3-yl)-urea," European Journal of Medicinal Chemistry, vol. 44, no. 11, pp. 4335-4343, 2009.

[21] S. L. Cao, Y. P. Feng, Y. Y. Jiang, S. Y. Liu, G. Y. Ding, and R. T. Li, "Synthesis and in vitro antitumor activity of $4(3 \mathrm{H})$ quinazolinone derivatives with dithiocarbamate side chains," Bioorganic and Medicinal Chemistry Letters, vol. 15, no. 7, pp. 1915-1917, 2005.

[22] A. M. Al-Obaid, S. G. Abdel-Hamide, H. A. El-Kashef et al., "Substituted quinazolines, part 3. Synthesis, in vitro antitumor activity and molecular modeling study of certain 2-thieno4(3H)-quinazolinone analogs," European Journal of Medicinal Chemistry, vol. 44, no. 6, pp. 2379-2391, 2009.

[23] E. Bansal, V. K. Srivastava, and A. Kumar, "Newer substituted $\beta$-aminonaphthalenes as potent anti-inflammatory agents," Arzneimittel-Forschung/Drug Research, vol. 50, no. 11, pp. 1009-1014, 2000.

[24] S. K. Srivastava, S. L. Srivastava, and S. D. Srivastava, "Synthesis of new 2-chlorophenothiazinothiadiazol-2-oxoazetidines: antimicrobial and antiinflammatory agents," Indian Journal of Chemistry B, vol. 39, no. 6, pp. 464-467, 2000.

[25] A. Kumar, B. P. Jaju, and J. N. Sinha, "1-(2-carboxyhenyl)3-chloro-4-aryl-azetidin-2-ones as potent anti-inflammatory agents," Indian Journal of Pharmaceutical Sciences, vol. 52, no. 6, pp. 257-260, 1990.

[26] R. Yadav, S. D. Srivastava, and S. K. Srivastava, "Synthesis, antimicrobial and antiinflammatory activities of 4oxothiazolidines and their 5-arylidenes," Indian Journal of Chemistry B, vol. 44, no. 6, pp. 1262-1266, 2005.

[27] B. Goel, T. Ram, R. Tyagi et al., "2-Substituted-3-(4-bromo-2carboxyphenyl)-5-methyl-4-thiazolidinones as potential antiinflammatory agents," European Journal of Medicinal Chemistry, vol. 34, no. 3, pp. 265-269, 1999.

[28] M. T. Bogert, "Researches on quinazolines (eighteenth paper), on 2,3-dialkyl-4-quinazolones and the products obtained by 
alkylating 2-alkyl-4-quinazolones (2-alkyl-4-hydroxy quinazolones)," Journal of the American Chemical Society, vol. 29, no. 4, pp. 517-536, 1907.

[29] C. A. Winter, E. A. Risley, and G. W. Nuss, "Carrageenan induced oedema in hind paw of the rat an as assay for antiinflammatory drugs," Proceedings of the Society for Experimental Biology and Medicine, vol. 111, pp. 544-550, 1962.

[30] B. A. Berkowitz, A. D. Finck, and S. H. Ngai, "Nitrous oxide analgesia: reversal by naloxone and development of tolerance," Pharmacology and Experimental Therapeutics, vol. 203, pp. 539-547, 1977. 

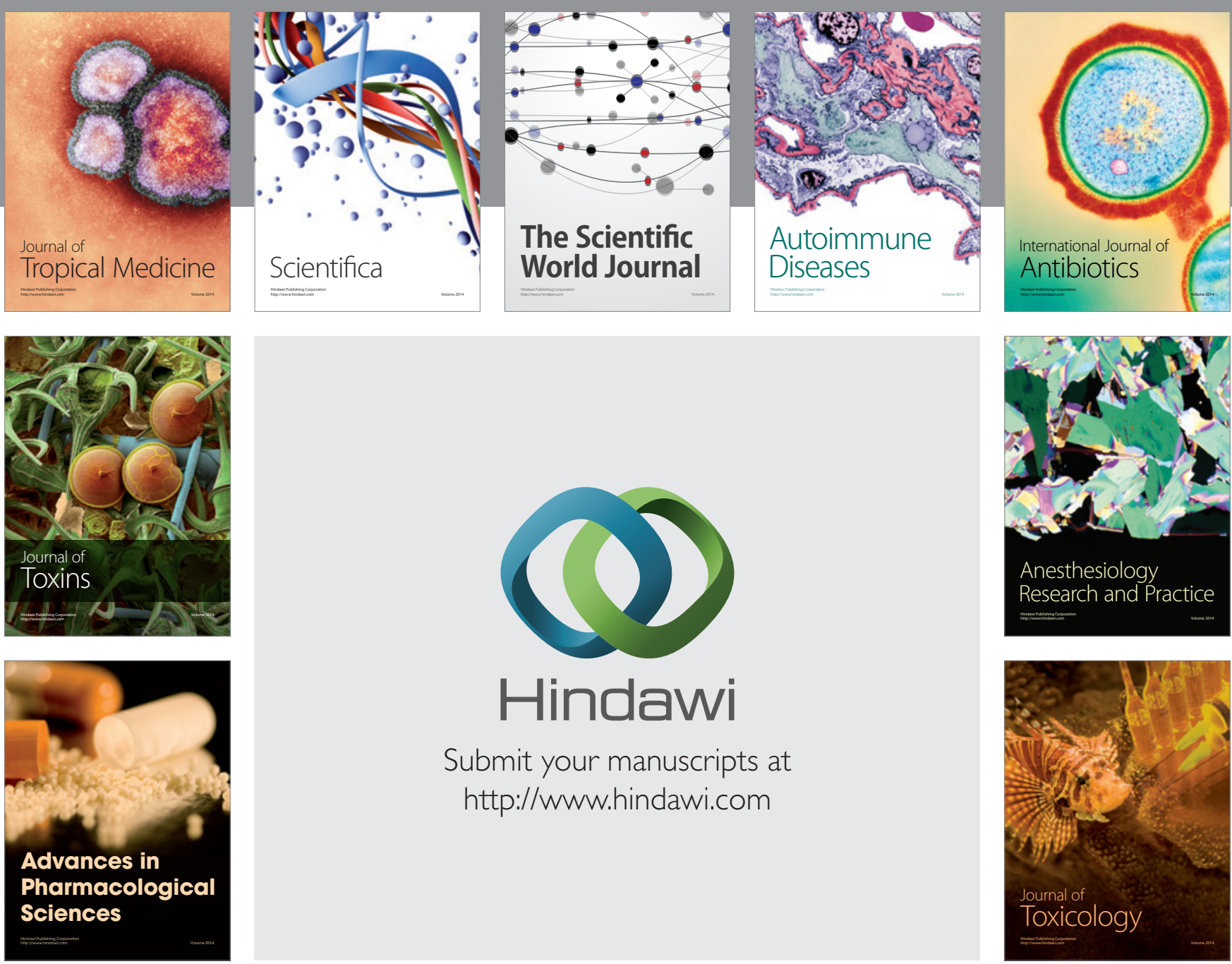

\section{Hindawi}

Submit your manuscripts at

http://www.hindawi.com
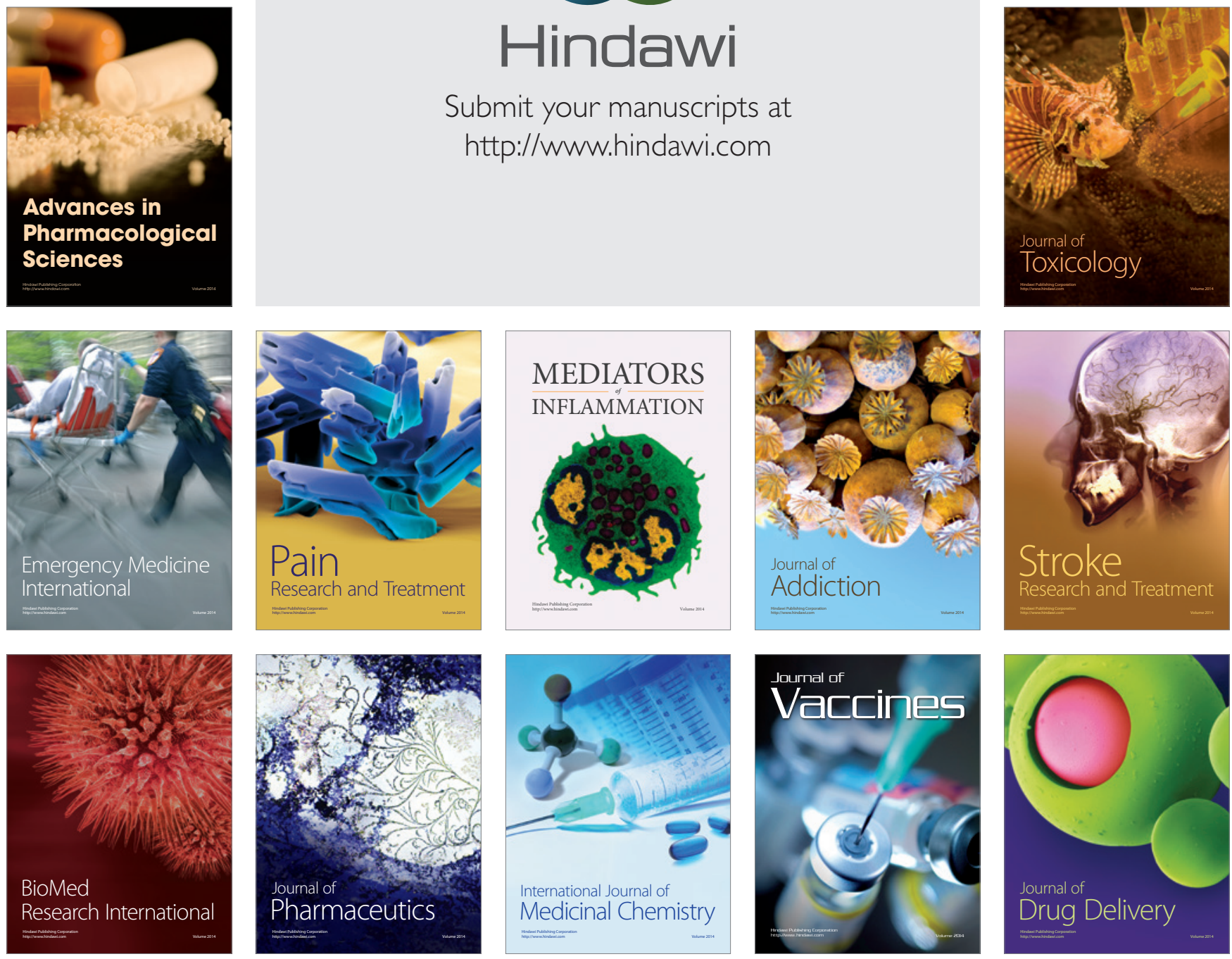